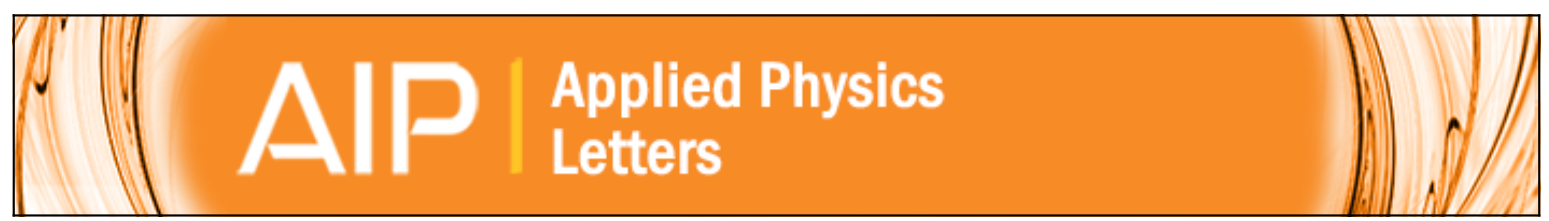

\title{
Method to calculate electric fields at very small tip-sample distances in atomic force microscopy
}

G. M. Sacha

Citation: Applied Physics Letters 97, 033115 (2010); doi: 10.1063/1.3467676

View online: $\mathrm{http}: / / \mathrm{dx}$. doi.org/10.1063/1.3467676

View Table of Contents: http://scitation.aip.org/content/aip/journal/apl/97/3?ver=pdfcov

Published by the AIP Publishing

\section{Articles you may be interested in}

An alternative method to determining optical lever sensitivity in atomic force microscopy without tip-sample contact

Rev. Sci. Instrum. 81, 073711 (2010); 10.1063/1.3459886

Tip-sample distance control using photothermal actuation of a small cantilever for high-speed atomic force microscopy

Rev. Sci. Instrum. 78, 083702 (2007); 10.1063/1.2766825

Tuning the TipSample Forces in Dynamic Atomic Force Microscopy

AIP Conf. Proc. 696, 377 (2003); 10.1063/1.1639721

Atomic force microscopy-induced electric field in ferroelectric thin films

J. Appl. Phys. 94, 4053 (2003); 10.1063/1.1603345

A direct method to calculate tip-sample forces from frequency shifts in frequency-modulation atomic force microscopy

Appl. Phys. Lett. 78, 123 (2001); 10.1063/1.1335546

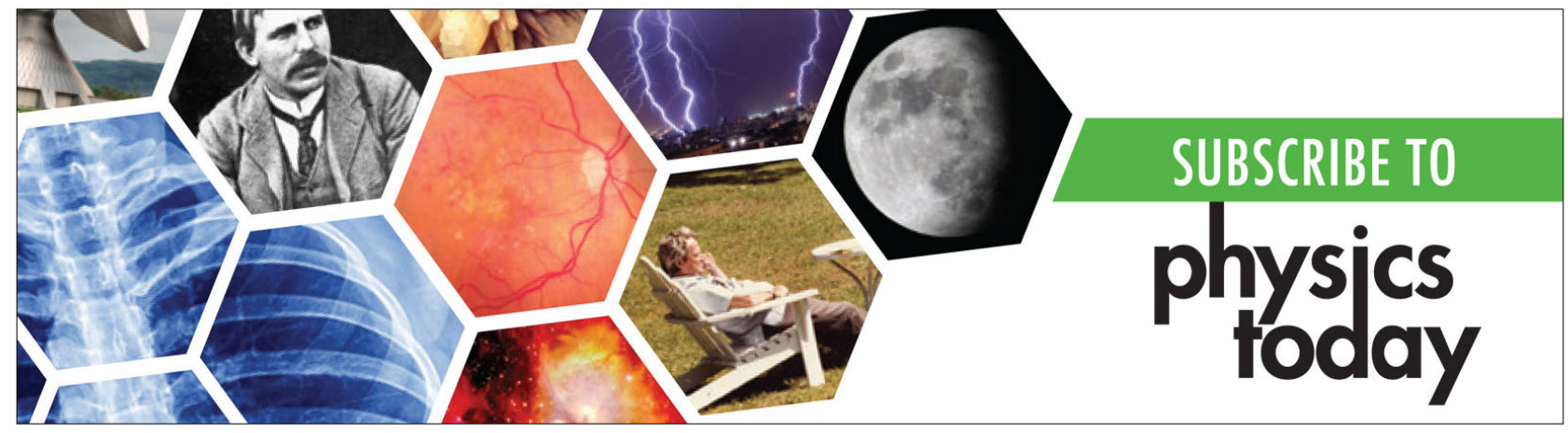




\title{
Method to calculate electric fields at very small tip-sample distances in atomic force microscopy
}

\author{
G. M. Sacha ${ }^{a}$ \\ Departamento de Ingeniería Informática, Grupo de Neurocomputación Biológica, Universidad Autónoma \\ de Madrid, Cantoblanco, Madrid E-28049, Spain
}

(Received 9 June 2010; accepted 1 July 2010; published online 23 July 2010)

\begin{abstract}
A method to calculate electric magnitudes at very small tip-sample distances in atomic force microscopy is presented. We show that the method accurately calculates the electrostatic potential and vertical force for electrostatic force microscopy geometries that cannot be correctly simulated by the standard techniques. This technique can accurately calculate tip-sample distances four orders of magnitude smaller than the tip radius. We also demonstrate that, at this range, traditional techniques underestimate the electrostatic force in almost 30\%. Finally, we calculate the jump-to-contact distance for geometries obtained from experiments that combine atomic force microscopy and scanning tunneling microscopy. (C) 2010 American Institute of Physics.
\end{abstract}

[doi:10.1063/1.3467676]

When electrostatic force microscopy (EFM) (Refs. 1-7) is working at the nanoscale, several interacting parameters have a strong influence in the signal. However, since EFM is a nonlocal technique due to the long-range nature of the electric interaction, there is no simple way to directly relate the EFM images with the microscope setup ${ }^{8}$ or the dielectric and topographic properties of the sample. ${ }^{9}$ One of the most important problems that must be faced when the influence of the microscope setup is going to be taken into account ${ }^{10}$ is the different scale of some magnitudes such as the tip length or the tip-sample distance. The image charge method $^{11,12}$ deals with this problem by replacing the surface charge on the metallic objects by a set of charge elements inside them. Although this strategy has given good results in many cases, the difference between the relevant distances of the system is growing every day since the experimental and technological worlds are increasing the precision of their applications.

In this article we propose a method that is not affected by the different order of magnitude of the relevant distances. We also analyze traditional methods and demonstrate that they cannot accurately simulate systems where the distances differs more than three orders of magnitude. Finally, we use the method to calculate the jump-to-contact (JTC) distance for relatively stiff cantilevers, which are able to work at distances as small as a few Armstrongs. These results can be used to estimate the best experimental setup in recently developed techniques that combines atomic force microscopy (AFM) and scanning tunneling microscopy (STM).

In a typical EFM setup, we have a metallic tip connected to a battery that applies a constant electric potential $V_{0}$. The tip is placed over a sample at a tip-sample distance D. To solve electrostatic problems with this geometry, an algorithm called the generalized image charge method (Ref. 13) (GICM) has been developed. The GICM replaces the surface charge density by a set of charges inside the metallic tip. The values $q_{i}$, positions $r_{i}$ and number of charges $N_{C}$ are not known and must be estimated individually every time the geometry changes. The standard minimization technique used by the GICM has been described elsewhere ${ }^{14}$ and its efficiency has

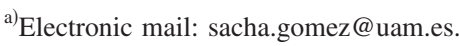

been demonstrated for systems where the sample includes objects such as carbon nanotubes ${ }^{15}$ or graphene. ${ }^{16}$ The tip is characterized by three geometrical parameters: The apex radius $\mathrm{R}$, the half-angle $\theta$ and the length $\mathrm{L}$. In the standard approximation, the tip surface is discretized by a set of points $N_{P}$ divided in three regions: the apex (region 1), the cone (region 2), and the rounded end of the tip (region 3). The sum of the points $N_{P}$ is obtained from the three regions $\left(N_{P}=N_{1}+N_{2}+N_{3}\right)$. A common feature of this method is that it gives the same importance to the error obtained at any point of the surface. However, the error at the tip apex $\left(N_{1}\right)$ is most important than the one obtained from the points at the conical region $\left(N_{2}\right)$ since the tip apex is the region where the electrostatic interaction is stronger. This problem is typically solved by increasing the weight of the points at the tip apex by changing the proportion between $N_{1}$ and $N_{2}$. However, using this strategy the computation time is strongly increased. Moreover, sometimes the minimization adjusts well the electrostatic potential for $N_{1}$ and almost forgets $N_{2}$ and $N_{3}$ because of the much bigger weight of $N_{1}$.

To deal with these problems, we have developed a minimization strategy, called GICM-fixed $\left(\mathrm{GICM}_{\mathrm{f}}\right)$, that is able to accurately calculate the electrostatic potential around the shorter distance of the system. The technique uses one of the parameters $q_{i}$ to fix the electrostatic potential at the lowest point (shorter distance) of the tip apex: $x_{1}=(x=0, z=D)$. To be sure that the boundary condition $V\left(x_{1}\right)=V_{0}$ is perfectly fitted, we can write the first charge/coefficient $\left(q_{1}\right)$ as a function of the others $\left(q_{2}\right.$ to $\left.q_{N_{c}}\right)$ in the following form:

$$
q_{1}=\frac{V_{0}-\Sigma_{i=2}^{N_{C}} q_{i} F_{i}(x=0, \quad z=D)}{F_{1}(x=0, \quad z=D)},
$$

where $x$ is the radial coordinate, $z$ the vertical one, and $F$ the Green function of the i-charge element. Technical details about the Green Function equations, boundary conditions, and electrostatic potential solution can be found in Sacha et al. ${ }^{13}$

Including this modification in the electrostatic potential, the least-square minimization process can be applied using the following expression: 


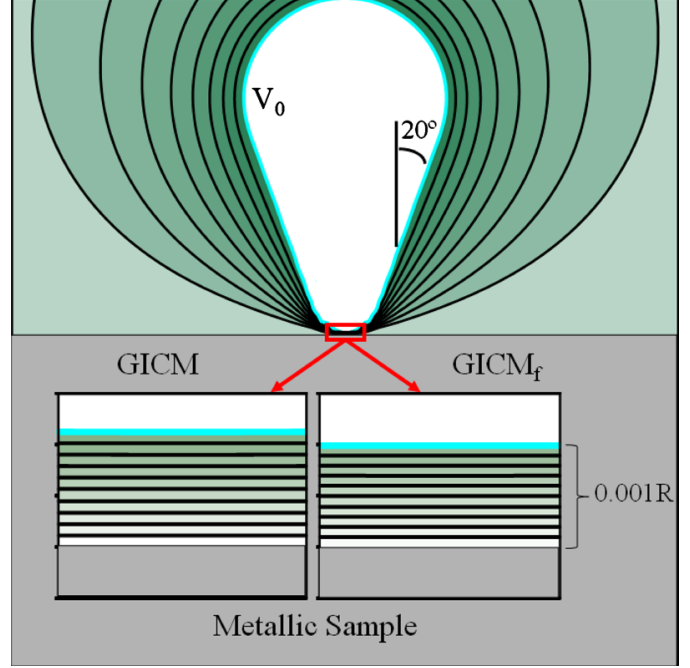

FIG. 1. (Color online) Equipotential lines between an electrostatic force microscope tip and a metallic sample when the tip-sample distance is 0.001 smaller than the tip radius. Two different scales are shown. The smallest one has been obtained both for the standard GICM minimization and the $\mathrm{GICM}_{\mathrm{f}}$ modification.

$$
\begin{aligned}
\chi^{2}= & \sum_{j=1}^{N_{P}}\left[F_{1}\left(x_{i}\right) \frac{V_{i}-\sum_{i=2}^{N_{C}} q_{i} F_{i}(x=0, \quad z=D)}{F_{1}(x=0, \quad z=D)}\right. \\
& \left.+\sum_{i=2}^{N_{C}} q_{i} F_{i}\left(x_{i}\right)-V_{j}\right]^{2} .
\end{aligned}
$$

Applying the minimization routine to $\chi^{2}$, we obtain the following set of $k$ equations for the coefficients $q_{2}$ to $q_{N_{c}}$ :

$$
\begin{gathered}
\sum_{j=1}^{N_{P}} \sum_{i=2}^{N_{C}} q_{i} S_{j}\left[F_{i}\left(x_{j}\right) F_{1}\left(x_{1}\right)-F_{i}\left(x_{1}\right) F_{1}\left(x_{i}\right)\right] \\
=V_{0} \sum_{j=1}^{N_{P}} S_{j}\left[F_{1}\left(x_{1}\right)-F_{1}\left(x_{j}\right)\right],
\end{gathered}
$$

where $S_{j}=F_{k}\left(x_{j}\right) F_{1}\left(x_{1}\right)-F_{1}\left(x_{j}\right) F_{k}\left(x_{1}\right)$ and $x_{1}=(x=0, z=D)$. The result of this calculation gives the best values for $q_{i}$ with $i=\left\{2, N_{C}\right\}\left[q_{I}\right.$ is obtained from Eq. (1)]. In Fig. 1 we show the electrostatic potential for a tip $\left(\theta=20^{\circ}, L=10 \mathrm{R}\right)$ over a metallic sample at $D=10^{-3} \mathrm{R}$. The figure shows a couple of zoomed images of the tip-apex region. In the closest one, we can see the difference between the standard GICM minimization and the GICM $_{\mathrm{f}}$. The $\mathrm{GICM}_{\mathrm{f}}$ perfectly fits the equipotential $V=V_{0}$ at $D=10^{-3}$ R. The GICM, however, places $V=V_{0}$ at a higher distance. In practical terms, this can be interpreted as a tip that is placed at $D>10^{-3} \mathrm{R}$. Because of this effect, the electric field and all the physical magnitudes related to the electrostatic potential are smaller in absolute value.

In Figs. 2 we explicitly show the difference in the electrostatic potential drop at the tip apex between the GICM and $\mathrm{GICM}_{\mathrm{f}}$. Due to the wrong solution obtained by the GICM, the electrostatic potential only changes $0.89 V_{0}$ between the metallic surface and $D=10^{-3} \mathrm{R}$. The electrostatic potential difference is exactly $V_{0}$ for the $\operatorname{GICM}_{\mathrm{f}}$. In this region, the electric field can be considered constant $\left(E=V_{0} / D\right)$ since the geometry converges to a parallel plate capacitor when $D$ $\rightarrow 0$. In the case under study we have $E(\mathrm{GICM})=890 \mathrm{~V} / \mathrm{R}$ instead of the correct value $E\left(\mathrm{GICM}_{\mathrm{f}}\right)=1000 \mathrm{~V} / \mathrm{R}$. Both for the electrostatic potential and electric field, the real value is

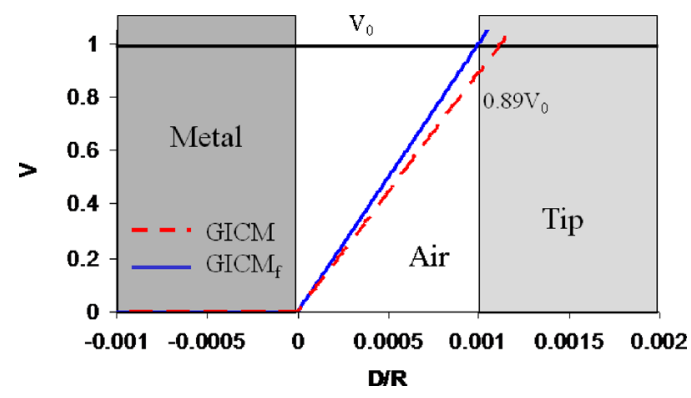

FIG. 2. (Color online) Electrostatic potential fall between the tip and a metallic sample along the tip axis for $D=0.001 \mathrm{R}, L=10 \mathrm{R}$, and $\theta=20^{\circ}$.

around $10 \%$ higher than the one obtained by the GICM. This mistake is directly translated to measurable magnitudes such us the vertical force between tip and sample. In Fig. 3 we show the normalized force versus $\mathrm{D} / \mathrm{R}$ obtained both for the GICM and the GICM $_{\mathrm{f}}$. The difference between $F(\mathrm{GICM})$ and $F\left(\mathrm{GICM}_{\mathrm{f}}\right)$ increases when D/R decreases. For the smallest distance that $\mathrm{F}$ can be obtained before the solution becomes unstable $(D / R=0.0007), \mathrm{F}(\mathrm{GICM})$ is $27.5 \%$ smaller than $F\left(\mathrm{GICM}_{\mathrm{f}}\right)$.

The $\mathrm{GICM}_{\mathrm{f}}$ includes a modification of the standard leastsquares minimization technique that can be easily extrapolated to different numerical methods such as the one proposed by Kalinin et al. ${ }^{17}$ and Morozovska et al. ${ }^{18}$ In these articles, several charged elements such as disks, lines or punctual charges are used to obtain the electrostatic potential around the tip. As well as the standard GICM, this configuration can be combined with the $\mathrm{GICM}_{\mathrm{f}}$ minimization to improve the quality of the results around the tip apex when $\mathrm{D}$ is very small.

Recently, a technique ${ }^{19}$ that combines AFM and STM has been used to characterize the dipole moment at monoatomic steps. This kind of techniques works at a very small tip-sample distance. The mechanical instability that induces the JTC (Ref. 20) is avoided using relatively stiff cantilevers. In Fig. 4(a) we use GICM $_{\mathrm{f}}$ simulations to calculate the JTC distance and the real position of the tip apex by comparing the absolute value of the electrostatic force with the force applied by the spring constant of the cantilever. We have used the parameters of the experimental results by Park et $a l .{ }^{19}$ For $K=88 \mathrm{~N} / \mathrm{m}$, the JTC distance is $3 \AA$, which makes the tip apex been at $1.5 \AA$ from the surface. In Fig. 4(b) we show the JTC and tip apex distances for different spring constants. As we can see, both distances exponentially increase when $\mathrm{K}$ decreases. This figure can be useful for the experimentalists to estimate the spring constant that must be

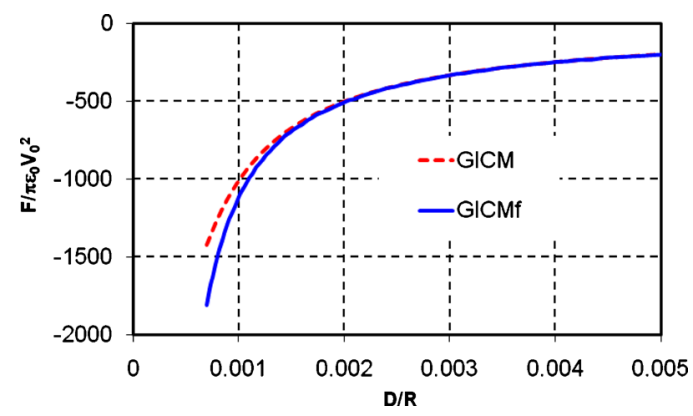

FIG. 3. (Color online) Normalized electrostatic force for both the GICM and the GICM $D=0.001$ R, $L=10$ R, and $\theta=20^{\circ}$ 
(a)

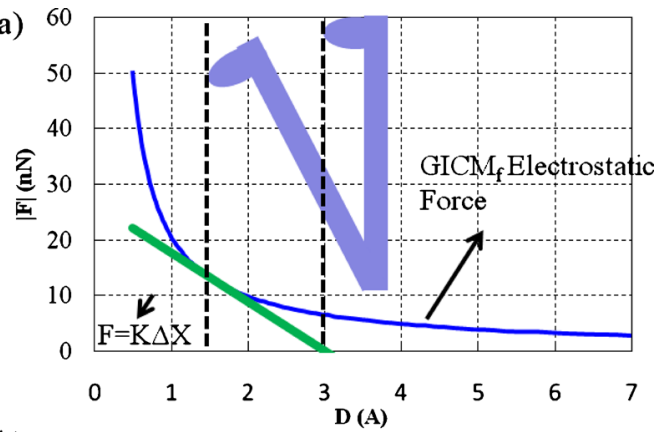

(b)

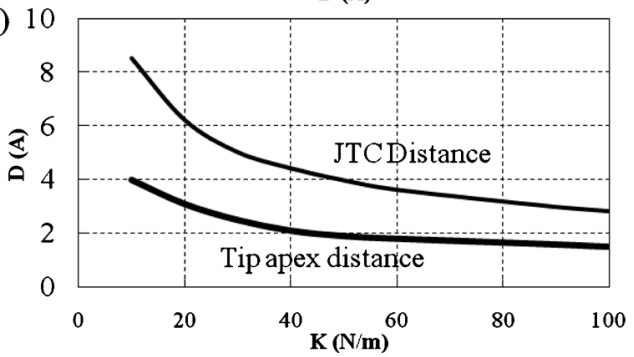

FIG. 4. (Color online) (a) Spring and $\mathrm{GICM}_{\mathrm{f}}$ electrostatic force for a tip placed at $D=3 \AA$ and $K=88 \mathrm{~N} / \mathrm{m}$. (b) JTC and Tip-apex distances for different spring constants, $R=70 \mathrm{~nm}$.

used to work at very small tip-sample distances in AFMSTM experiments.

In conclusion, we have developed a method to calculate electric magnitudes such as the electrostatic potential, the electric field or the electrostatic force for EFM geometries with magnitudes that differs more than three orders of magnitude. As an example, we correctly calculate the vertical electric force between an EFM tip and a metallic sample with a separation between them almost four times smaller than the tip radius. At the closest tip-sample distance, the difference in the electric force between our technique and the GICM (a technique that is already optimized for geometries that differs several orders of magnitude) is almost $30 \%$. We have used $\mathrm{GICM}_{\mathrm{f}}$ simulations to estimate the spring constant that must be used in experiments where the tip-sample distance must be as small as a few Armstrongs.
Author acknowledges J. J. Sáenz, P. Varona, E. Serrano, and F. Rodríguez for insightful discussions. This work was supported by Ministerio de Educación y Ciencia Grant Nos. TIN 2007-65989 and Comunidad Autonoma de Madrid S-SEM-0255-2006.

${ }^{1}$ S. Guriyanova, D. S. Golovko, and E. Bonaccurso, Meas. Sci. Technol. 21, 025502 (2010).

${ }^{2}$ A. Verdaguer, M. Cardellach, J. J. Segura, G. M. Sacha, J. Moser, M. Zdrojek, A. Bachtold, and J. Fraxedas, Appl. Phys. Lett. 94, 233105 (2009)

${ }^{3}$ C. Riedel, R. Arinero, Ph. Tordjeman, M. Ramonda, G. Lévêque, G. A. Schwartz, D. G. de Oteyza, A. Alegría, and J. Colmenero, Eur. Phys. J.: Appl. Phys. 50, 10501 (2010).

${ }^{4}$ J. Hu, X.-D. Xiao, and M. Salmeron, Appl. Phys. Lett. 67, 476 (1995).

${ }^{5}$ A. N. Morozovska, E. A. Eliseev, and S. V. Kalinin, J. Appl. Phys. 102, 074105 (2007).

${ }^{6}$ E. Palacios-Lidón, J. Abellán, J. Colchero, C. Munuera, and C. Ocal, Appl. Phys. Lett. 87, 154106 (2005).

${ }^{7}$ S. F. Lyuksyutov, R. A. Vaia, P. B. Paramonov, S. Juhl, L. Waterhouse, R. M. Ralich, G. Sigalov, and E. Sancaktar, Nature Mater. 2, 468 (2003).

${ }^{8}$ G. M. Sacha and J. J. Sáenz, Appl. Phys. Lett. 85, 2610 (2004).

${ }^{9}$ S. Gómez-Moñivas, J. J. Sáenz, R. Carminati, and J. J. Greffet, Appl. Phys. Lett. 76, 2955 (2000).

${ }^{10}$ A. Gomez, A. Avila, and J. P. Hinestroza, J. Electrost. 68, 79 (2010).

${ }^{11}$ F. Kung and H. T. Chuah, PIER 42, 49 (2003).

${ }^{12}$ Y.-L. Li, J.-Y. Huang, and M.-J. Wang, PIER 81, 343 (2008).

${ }^{13}$ G. M. Sacha, E. Sahagún, and J. J. Sáenz, J. Appl. Phys. 101, 024310 (2007).

${ }^{14}$ G. M. Sacha, F. Rodríguez, E. Serrano, and P. Varona, J. of Electromagnetic Waves and Applications 24, 1145 (2010).

${ }^{15}$ G. M. Sacha, C. Gómez-Navarro, J. J. Sáenz, and J. Gómez-Herrero, Appl. Phys. Lett. 89, 173122 (2006).

${ }^{16}$ G. M. Sacha, M. Cardellach, J. J. Segura, J. Moser, A. Bachtold, J. Fraxedas, and A. Verdaguer, Nanotechnology 20, 285704 (2009).

${ }^{17}$ S. V. Kalinin, S. Jesse, B. J. Rodriguez, E. A. Eliseev, V. Gopalan, and A. N. Morozovska, Appl. Phys. Lett. 90, 212905 (2007).

${ }^{18}$ A. N. Morozovska, E. A. Eliseev, G. S. Svechnikov, V. Gopalan, and S. V. Kalinin, J. Appl. Phys. 103, 124110 (2008).

${ }^{19}$ J. Y. Park, G. M. Sacha, M. Enachescu, D. F. Ogletree, R. A. Ribeiro, P. C. Canfield, C. J. Jenks, P. A. Thiel, J. J. Saenz, and M. Salmeron, Phys. Rev. Lett. 95, 136802 (2005).

${ }^{20}$ G. M. Sacha, A. Verdaguer, and M. Salmeron, J. Phys. Chem. B 110, 14870 (2006). 